\title{
On infinite-finite duality pairs of directed graphs
}

\author{
Péter L. Erdôs ${ }^{\mathrm{a}, 1}$, Claude Tardif ${ }^{\mathrm{b}, 2}$, Gábor Tardos ${ }^{\mathrm{c}, \mathrm{a}, 3}$ \\ ${ }^{a}$ Alfréd Rényi Institute of Mathematics, Reáltanoda u 13-15 Budapest, 1053 Hungary \\ email: <elp,tardos>@renyi.hu \\ ${ }^{b}$ Royal Military College of Canada, PO Box 17000 Station "Forces" \\ Kingston, Ontario, Canada, K7K $7 B 4$ \\ email: Claude.Tardif@rmc.ca \\ ${ }^{c}$ School of Computing Science, Simon Fraser University \\ 8888 University Drive, Burnaby, BC, Canada, V5A $1 S 6$
}

\begin{abstract}
The $(\mathcal{A}, \mathcal{D})$ duality pairs play a crucial role in the theory of general relational structures and in Constraint Satisfaction Problems. The case where both sides are finite is fully characterized. The case where both sides are infinite seems to be very complex. It is also known that no finite-infinite duality pair is possible if we make the additional restriction that both classes are antichains. In this paper (which is the first one of a series) we start the detailed study of the infinite-finite case.

Here we concentrate on directed graphs. We prove some elementary properties of the infinite-finite duality pairs, including lower and upper bounds on the size of $\mathcal{D}$, and show that the elements of $\mathcal{A}$ must be equivalent to forests if $\mathcal{A}$ is an antichain. Then we construct instructive examples, where the elements of $\mathcal{A}$ are paths or trees. Note that the existence of infinite-finite antichain dualities was not previously known.
\end{abstract}

Keywords: graph homomorphism; duality pairs; general relational structures; Constraint Satisfaction Problem; regular languages; nondeterministic finite automaton;

\section{Introduction}

In this paper we consider directed graphs, homomorphisms between them, and especially duality pairs. We start with the definitions.

A directed graph $G$ is a pair $(V, E)$ with $V=V(G)$ the set of vertices and $E=$ $E(G) \subseteq V^{2}$ the set of (directed) edges. Unless stated otherwise "graph" refers to finite directed graphs in this paper. Forgetting about the orientation of the edges one

\footnotetext{
${ }^{1}$ Research supported in part by the Hungarian NSF, under contract NK 78439 and K 68262

${ }^{2}$ Research supported by grants from NSERC and ARP.

${ }^{3}$ Research supported in part by an NSERC Discovery grant and by the Hungarian OTKA grants T-046234, AT048826 and NK-62321
} 
gets the underlying undirected graph. For simplicity we drop the term "oriented" when referring to (oriented) paths, (oriented) trees and (oriented) forests, these are (directed) graphs whose underlying undirected graphs are path, trees, respectively forests in the traditional sense. In particular, paths, trees and forests have no loops and no pair of vertices is connected in both directions. Similarly, when we call a graph connected, refer to the connected components or the girth of a graph or to a cycle in a graph we mean the corresponding notion in the underlying undirected graph.

A homomorphism $f$ between graphs $G$ and $H$ is a map $f: V(G) \rightarrow V(H)$ satisfying that for every edge $(x, y) \in E(G)$ we have $(f(x), f(y)) \in E(H)$. We write $f: G \rightarrow H$ to express that $f$ is a homomorphism from $G$ to $H$ and we write $G \rightarrow H$ to express that such a homomorphism exists. This is clearly a transitive and reflexive relation. We write $G \not \rightarrow H$ if no homomorphism from $G$ to $H$ exists and call a family of graphs an antichain if no homomorphism exists between any two distinct members.

If both $G \rightarrow H$ and $H \rightarrow G$ hold for a pair of graphs we say $G$ and $H$ are equivalent. This is clearly an equivalence relation. In any equivalence class the graph with the fewest vertices is unique up to isomorphism. We call such a graph a core and also the core of any graph in its equivalence class. It is easy to see that a graph $G$ is a core if and only if every homomorphism $f: G \rightarrow G$ is an isomorphism.

We say a graph $G$ is minimal in a family $\mathcal{A}$ of graphs if $G \in \mathcal{A}$ and any graph $H \in \mathcal{A}$ satisfying $H \rightarrow G$ is equivalent to $G$. We define the dual notion of maximal in a family of graphs similarly, but with the homomorphism condition reversed. Note that there are two-way infinite chains of graphs, so infinite classes do not always have minimal or maximal elements.

A duality pair is a pair $(\mathcal{A}, \mathcal{D})$ of families of graphs satisfying that for every graph $G$ we have either $A \rightarrow G$ for some $A \in \mathcal{A}$ or $G \rightarrow D$ for some $D \in \mathcal{D}$ but not both. If $(\mathcal{A}, \mathcal{D})$ is a duality pair we call $\mathcal{D}$ a dual of $\mathcal{A}$. Note, however, that this relation is not symmetric. Duality pairs were first introduced in [6] and investigated in detail in [7] for the special case when $|\mathcal{A}|=1$.

Clearly, each graph in $\mathcal{A}$ and $\mathcal{D}$ in a duality pair $(\mathcal{A}, \mathcal{D})$ can be replaced with its core to obtain another duality pair $\left(\mathcal{A}^{\prime}, \mathcal{D}^{\prime}\right)$ so we can (and often will) assume that both sides of a duality pair consist of cores. Further if $A \rightarrow A^{\prime}$ with $A \neq A^{\prime}$ and $A, A^{\prime} \in \mathcal{A}$ we can remove $A^{\prime}$ from $\mathcal{A}$ without ruining the duality pair property. This way, if $\mathcal{A}$ is finite we can replace it with the antichain $\mathcal{A}^{\prime}$ of its minimal elements and the resulting pair $\left(\mathcal{A}^{\prime}, \mathcal{D}\right)$ is still a duality pair. Similarly, if $\mathcal{D}$ is finite we can replace it with the set $\mathcal{D}^{\prime}$ of its maximal elements to obtain a duality pair $\left(\mathcal{A}, \mathcal{D}^{\prime}\right)$ with $\mathcal{D}^{\prime}$ being an antichain. Note, however, that such transformation is not possible in general for infinite families.

It is a trivial observation that any family $\mathcal{A}$ has a dual set $\mathcal{D}$, simply take $\mathcal{D}=$ $\{G \mid \nexists A \in \mathcal{A}: A \rightarrow G\}$. For any family $\mathcal{D}$ of graphs one can similarly set $\mathcal{A}=\{G \mid \nexists D \in \mathcal{D}: G \rightarrow D\}$ making $(\mathcal{A}, \mathcal{D})$ a duality pair. Because of this abundance it is not reasonable to hope for a meaningful characterization of all 
duality pairs. But characterization of restricted classes of duality pairs have been done successfully already.

Theorem 1.1 ([7]). Each tree $T$ has a well-defined, unique (up to equivalence) graph $D(T)$ making $(\{T\},\{D(T)\})$ a duality pair. In all singleton duality pairs $(\{A\},\{D\})$ the graph $A$ is equivalent to a tree.

Theorem 1.2 ([5]). For any given finite family $\mathcal{A}$ of forests there exists a unique (up to the equivalence) antichain dual $\mathcal{D}(\mathcal{A})$ and it is finite. For any duality pair $(\mathcal{A}, \mathcal{D})$ with both $\mathcal{A}$ and $\mathcal{D}$ finite antichains all graphs $A \in \mathcal{A}$ are equivalent to forests.

We saw above that having antichains as the members of duality pairs can be considered as a relaxation of the finiteness condition. Having characterized the duality pairs with both sides finite it is natural to consider this relaxation. We start with quoting a result showing that there are probably too many infinite-infinite antichain duality pairs for a meaningful characterization.

Theorem 1.3 ([1]). Each finite antichain $\mathcal{A}$ of graphs that is not maximal can be extended

(i) to a duality pair $(\mathcal{B}, \mathcal{C})$ such that $\mathcal{A} \subset \mathcal{B}$ and both $\mathcal{B}$ and $\mathcal{C}$ are infinite antichains;

(ii) to a maximal infinite antichain, which is not a union of the sides of any duality pair.

This naturally leads to the question of finding or characterizing antichain duality pairs with one side finite while the other is an infinite antichain. Erdôs and Soukup [2] proved that no finite-infinite antichain duality pair exists and asked if infinitefinite ones do.

Theorem 1.4 ([2]). There exists no duality pair $(\mathcal{A}, \mathcal{D})$ with $\mathcal{A}$ finite and $\mathcal{D}$ an infinite antichain.

In this paper we answer the question of Erdős and Soukup by giving several examples of infinite-finite antichain duality pairs and also study what families can appear in the left side of such a duality pair. The final answer (a characterization of such families) will follow from the upcoming paper [4] that studies the problem in the more general context of relational structures.

In Section 2 we limit the complexity of any graph appearing in an antichain with a finite dual: it must be equivalent to a forest. For finite antichains this is implied by Theorem 1.2. We also show that such a family has to have bounded maximum degree and bounded number of components.

When the forests in a duality pair have only one component and maximum degree two we deal with families of paths. In Section 3 we exhibit specific infinite antichains of paths, some with, and some without a finite dual. 
In Section 4 we give a simple transformation turning the duality pairs in Section 3 into ones with non-path trees on the left side. However, these trees are still close to paths. We also give examples of infinite-singleton duality pairs where the left side consists of more complex trees constructed from arbitrary binary trees. One of these examples has an antichain on the left side. In another example of an infinite-finite antichain duality pair the left side consists of forests with several components.

\section{Why forests?}

In this section we prove that all graphs in the left side of an infinite-finite antichain duality pair must be equivalent to forests. This is an extension of the corresponding result for finite antichains in Theorem 1.2.

Theorem 2.1. Let $(\mathcal{A}, \mathcal{D})$ be a duality pair, where $\mathcal{D}$ is finite and $\mathcal{A}$ consists of cores. Then for each graph $A \in \mathcal{A}$ that is not a forest there exists another graph $B \in \mathcal{A}$ with $B \rightarrow A$ but $A \not \rightarrow B$.

This result can be proved from the Directed Sparse Incomparability Lemma, see $[1,2]$. We present a self contained proof instead.

Proof. Let $A \in \mathcal{A}$ be a graph that is not a forest. Let $(x, y)$ be an edge of $A$ contained in a cycle $C$. Let $A^{\prime}$ be the graph obtained from $A$ by removing this edge, adding a new vertex $x^{\prime}$ and the edge $\left(x^{\prime}, y\right)$. Notice that the map moving $x^{\prime}$ to $x$ and fixing all other vertices is an $A^{\prime} \rightarrow A$ homomorphism.

Let $X$ be an arbitrary tournament with more vertices than any of the (finitely many) graphs in $\mathcal{D}$. Let us consider the vertex set $V(X)$ (no edges yet) and disjoint copies $A_{u v}^{\prime}$ of $A^{\prime}$ for every edge $(u, v) \in E(X)$. We obtain the graph $Y$ by identifying the copy of $x$ in $A_{u v}^{\prime}$ with $u$ and the copy of $x^{\prime}$ in $A_{u v}^{\prime}$ with $v$ for all $(u, v) \in E(X)$.

Note that the natural $A^{\prime} \rightarrow A$ homomorphism can be applied to each copy $A_{u v}^{\prime}$ of $A^{\prime}$ as all the identified vertices are mapped to $x$. This gives us a natural homomorphism $g: Y \rightarrow A$.

As $(\mathcal{A}, \mathcal{D})$ is a duality pair we either have a graph $B \in \mathcal{A}$ with $B \rightarrow Y$ or a graph $D \in \mathcal{D}$ with $Y \rightarrow D$. In the latter case we have $|V(X)|>|V(D)|$, so by the pigeonhole principle we must have $f(u)=f(v)$ for an edge $(u, v) \in E(X)$. But this means that $f$ restricted to $A_{u v}^{\prime}$ is an $A \rightarrow D$ homomorphism, a clear contradiction. This leaves the former possibility only. We show that $B \in \mathcal{A}$ with $B \rightarrow Y$ satisfies the statement of the theorem.

Indeed we have $B \rightarrow Y \rightarrow A$. We will show $A \not \rightarrow Y$ and this implies $A \not \rightarrow B$. In the degenerate case when $A$ consists of a single loop edge $A \nrightarrow \rightarrow Y$ holds, since $Y$ is a tournament in this case. So we may assume $A$ is not a loop and as it is a core it does not even contain a loop. In particular $x \neq y$ and $C$ has length at least 2. Assume for a contradiction that a homomorphism $f: A \rightarrow Y$ exists. As $A$ is a core the homomorphism $f \circ g: A \rightarrow A$ must be an automorphism. Modifying $f$ 
appropriately, one can assume without loss of generality that $f \circ g$ is the identity, so $f(z) \in g^{-1}(z)$ for each vertex $z \in V(A)$. We must have $f(x) \in g^{-1}(x)=V(X)$ and $f(z) \notin V(X)$ for any other vertex $z$ of $A$. The vertices of the cycle $C$ except $x$ itself must be mapped in a single connected component of $Y \backslash V(X)$, in particular, in a single copy $A_{u v}^{\prime}$ of $A^{\prime}$. The image $f(y)$ of $y$ must be the copy of $y$ in $A_{u v}^{\prime}$, so to have $(f(x), f(y)) \in E(Y)$ we must have $f(x)=v$. This forces the image of the other edge incident to $x$ in the cycle $C$ outside $E(Y)$. The contradiction finishes the proof of the theorem.

Note that this theorem implies that if we have antichains in an infinite-finite duality pair of cores, then the left side contains forests only. Furthermore, something can be said without restricting attention to antichains. Let $(\mathcal{A}, \mathcal{D})$ be an infinitefinite duality pair of cores. We can remove from $\mathcal{A}$ all the graphs which are not forests but are "dominated" by one: the graphs $A \in \mathcal{A}$ for which a forest $B \in \mathcal{A}$ exists with $B \rightarrow A$, but $A$ itself is not an forest. Clearly, the set of graphs to which a homomorphism exists from a member of the remaining family $\mathcal{A}^{\prime}$ did not change, so $\left(\mathcal{A}^{\prime}, \mathcal{D}\right)$ is still a duality pair. This duality pair may still contain a graph $A \in \mathcal{A}^{\prime}$ that is not a forest, but such a graph must have infinitely many distinct graphs $B \in \mathcal{A}^{\prime}$ with $B \rightarrow A$. Indeed, if $A$ has only finitely many such dominating $B$, then any minimal graph in this finite set would violate the preceding theorem. From the Directed Sparse Incomparability Lemma one can also show that if a graph $A \in \mathcal{A}^{\prime}$ is imbalanced (containing a cycle with an unequal number of forward and reverse oriented edges), then the underlying undirected graphs of the graphs $B \in \mathcal{A}^{\prime}$ have unbounded girth.

In the following lemma we state the connection between having connected graphs on the left side of a duality pair and having a single graph on the right side. Recall that we call a graph connected if the underlying undirected graph is connected and use the term connected component in a similar way.

Lemma 2.2. Let $(\mathcal{A}, \mathcal{D})$ be an antichain duality pair with $\mathcal{A}$ consisting of cores. If a graph $A \in \mathcal{A}$ has $k$ connected components we have $|\mathcal{D}| \geq k$. But if all graphs in $\mathcal{A}$ are connected, then $|\mathcal{D}|=1$.

Proof. Take a graph $A \in \mathcal{A}$. Let $A_{1}, \ldots, A_{k}$ be the graphs obtained from $A$ by removing a single one of its $k$ components. For any $1 \leq i \leq k$ we have $A \not \rightarrow A_{i}$ (since $A$ is a core) furthermore we have $A_{i} \rightarrow A$, so as $\mathcal{A}$ is an antichain it contains no graph that has a homomorphism to $A_{i}$. As $(\mathcal{A}, \mathcal{D})$ is a duality pair each $A_{i}$ has a graph $D_{i} \in \mathcal{D}$ with $A_{i} \rightarrow D_{i}$. If we have $D_{i}=D_{j}$ for some $1 \leq i<j \leq k$ we can construct an $A \rightarrow D_{i}$ homomorphism by extending the $A_{i} \rightarrow D_{i}$ homomorphism to the missing component using the corresponding restriction of the $A_{j} \rightarrow D_{i}$ homomorphism. Since we must have $A \not \rightarrow D$ for $D \in \mathcal{D}$ all graphs $D_{i}$ are distinct and thus $|\mathcal{D}| \geq k$ as claimed.

Now assume that every $A \in \mathcal{A}$ is connected but still we have two graphs $D_{1} \neq D_{2}$ in $\mathcal{D}$. As $\mathcal{D}$ is an antichain the disjoint union $D$ of $D_{1}$ and $D_{2}$ does not have a homomorphism to any member of $\mathcal{D}$. By the duality pair property we must 
have a graph $A \in \mathcal{A}$ such that $A \rightarrow D$. As $A$ is connected this homomorphism maps $A$ either to $D_{1}$ or to $D_{2}$, giving $A \rightarrow D_{1}$ or $A \rightarrow D_{2}$, a contradiction.

An immediate corollary of this lemma is that if an antichain has a finite dual its members have a bounded number of components. For this we do not need the full strength of the antichain condition, it is enough to assume that we do not have a homomorphism between two graphs of $\mathcal{A}$ that avoids an entire connected component of the target graph. While replacing the left hand side of a duality pair with an equivalent antichain is not always possible, it is easy to see that replacing the left hand side of a duality pair with an equivalent family satisfying this constraint is always possible. If the right side is finite, then after this transformation the graphs in the left side have a bounded number of components.

We end this section by showing that the maximum degree is also bounded in an antichain of core graphs that has a finite dual.

Lemma 2.3. Let $(\mathcal{A}, \mathcal{D})$ be a duality pair with $\mathcal{A}$ an antichain consisting of cores and $\mathcal{D}$ finite. Any vertex of any graph $A \in \mathcal{A}$ has total degree (this is the sum of the in-degree and out-degree) at most $d_{0}=\sum_{D \in \mathcal{D}}|V(D)|$.

Proof. Let $A \in \mathcal{A}$ and $v \in V(A)$ and suppose the total degree $d$ of $v$ is larger than $d_{0}$. By Theorem $2.1 A$ is a forest, so $v$ cuts its component of $A$ into $d$ parts. Let us form the subgraphs $A_{1}, \ldots, A_{d}$ of $A$ by removing a single one of these parts from $A$. That is, each $A_{i}$ is obtained from $A$ by removing an edge $e$ from $A$ that connects $v$ to another vertex $w$ (with either orientation) and also removing the connected component of $w$ from the resulting graph. As $\mathcal{A}$ is an antichain of cores no member of $\mathcal{A}$ has a homomorphism to any of these subgraphs $A_{i}$, so by the duality pair property, there must be homomorphisms $f_{i}: A_{i} \rightarrow D_{i}$ from $A_{i}$ to certain graphs $D_{i} \in D$. From $d>d_{0}$ we must have $1 \leq i<j \leq d$ with $D_{i}=D_{j}$ and $f_{i}(v)=f_{j}(v)$. We construct an $A \rightarrow D_{i}$ homomorphism by extending $f_{i}$ with the restriction of $f_{j}$ to the part of $A$ missing from $A_{i}$. The contradiction finishes the proof.

\section{Antichains of paths}

In this section we give concrete examples of infinite antichains of paths with or without a finite dual. As paths are connected, Lemma 2.2 tells us that when looking for a finite dual it is always enough to consider duals consisting of a single graph.

To speak of (oriented) paths we use the natural correspondence between them and words over the binary alphabet $\{+,-\}$. We use standard notation with respect to these words, namely a word is a member of $\{+,-\}^{*}=\cup_{k>0}\{+,-\}^{k}$, where $\{+,-\}^{k}$ is the set of length $k$ sequences from the alphabet. For $x, y \in\{+,-\}^{*}$

and $k \geq 0$ we write $x y$ for the concatenation of $x$ and $y$ and $x^{k}$ for the word obtained by concatenating $k$ copies of $x$.

The correspondence is given by the map $p$ mapping $\{+,-\}^{*}$ to paths. For a word $x=x_{1} \ldots x_{k} \in\{+,-\}^{k}$ let $p(x)$ stand for the path consisting of $k$ edges 
with the $i$ 'th edge oriented forward if $x_{i}=+$ and backward otherwise. A bit informally we will refer to the first and last vertices of $p(x)$ in their obvious meaning, although formally the end vertices of the path $p(x)$ cannot be distinguished without knowing $x$. We say that a homomorphism $f: p(x) \rightarrow G$ maps $p(x)$ from $u$ to $v$ if the image of the first vertex of $p(x)$ is $u \in V(G)$ and the image of the last vertex is $v \in V(G)$. Note that although all (isomorphism classes of) paths will be obtained as images in this map the correspondence is not one-one: $p$ maps up to two distinct words to isomorphic paths (with the role of the first and last vertices reversed), for example $p(++-)$ and $p(+--)$ are isomorphic.

\subsection{Antichains without a finite dual}

The following observation is trivial: Take any infinite antichain of paths (as we will see such antichains are easy to find). The cardinality of the set of its subsets is continuum, and no two can have the same set for dual. Thus many have no finite dual, as the set of finite families of graphs is countable. This cardinality argument gives no explicit family without a finite dual. Here we set out to construct such a set.

Lemma 3.1. Let $G$ be a graph with $|V(G)| \leq k$ and assume that for some $x \in$ $\{+,-\}^{*}$ we have $p\left(x^{k}\right) \rightarrow G$. Then for each $\ell \geq 0$ we also have $p\left(x^{\ell}\right) \rightarrow G$.

Proof. There is nothing to prove if $x$ is the empty string. Otherwise, by considering the homomorphism $p\left(x^{k}\right) \rightarrow G$, one can find vertices $v_{0}, \ldots, v_{k}$ in $G$ such that for each $1 \leq i \leq k$ a suitable restriction of the homomorphism maps $p(x)$ from $v_{i-1}$ to $v_{i}$. By the pigeonhole principle we find $v_{i}=v_{j}$ for some $0 \leq i<j \leq k$. Thus, we can map the $p\left(x^{j-i}\right)$ to $G$ with both endpoints mapping to the same vertex. This closed walk can take the homomorphic image of $p\left(x^{\ell}\right)$ for any $\ell$.

Example 3.2. Let $Q_{k}=p\left(\left(+(+-)^{k}\right)^{k}++\right)$ and consider any infinite family $\mathcal{A} \subseteq\left\{Q_{k} \mid k \geq 1\right\}$. Then $\mathcal{A}$ is an antichain of paths and has no finite dual.

Proof. To see that $\mathcal{A}$ is an antichain observe that the height of a path defined as the maximal difference between the number of forward edges and the number of backward edges in a sub-path cannot be decreased by a homomorphism. As the height of $Q_{k}$ is $k+2$ we have $Q_{k} \not \rightarrow Q_{\ell}$ for $\ell<k$. But $p\left(+(+-)^{k}++\right)$ is a sub-path of $Q_{k}$ and even this sub-path does not map to $Q_{\ell}$ for $\ell>k$. A similar argument also shows that all $Q_{k}$ are cores: as deleting either the first or the last edge of $Q_{k}$ decreases its height any homomorphism $Q_{k} \rightarrow Q_{k}$ must be onto and thus an isomorphism.

Assume $(\mathcal{A}, \mathcal{D})$ is a duality pair. Let $Q_{k} \in \mathcal{A}$ and consider $Q_{k}^{\prime}=p\left(\left(+(+-)^{k}\right)^{k}\right)$. Clearly, $Q_{k}^{\prime} \rightarrow Q_{k}$, so we have $Q_{\ell} \not \rightarrow Q_{k}^{\prime}$ for $\ell \neq k$ by the antichain property and $Q_{k} \nrightarrow \rightarrow Q_{k}^{\prime}$ since $Q_{k}$ is a core. So we must have $D \in \mathcal{D}$ with $Q_{k}^{\prime} \rightarrow D$. We claim that $|V(D)|>k$. Indeed, otherwise by Lemma 3.1 we have $Q_{k} \rightarrow$ $p\left(\left(+(+-)^{k}\right)^{k+1}\right) \rightarrow D$, a contradiction. As $\mathcal{A}$ is infinite $k$ could be chosen arbitrarily large, so $\mathcal{D}$ must have arbitrarily large graphs and, thus, cannot be finite. 


\subsection{Infinite antichains of paths with a finite dual}

Our first infinite-finite antichain duality pair, the $s=3$ case of the next example, is the smallest possible such example in the sense that the dual is a single graph on four vertices, while no graph or family of graphs on fewer vertices is a dual of an infinite antichain.

Example 3.3. Let $P_{k}^{s}=p\left(+^{s}\left(-+^{s-1}\right)^{k}+\right)$ for $s \geq 1, k \geq 0$ and let $D_{s}$ be the graph obtained from the transitive tournament on $s+1$ vertices by deleting the edge connecting the source and the sink. Then $\left(\left\{P_{k}^{s} \mid k \geq 0\right\},\left\{D_{s}\right\}\right)$ is an antichain duality pair of cores for $s \geq 3$.

Proof. To see that the infinite side is an antichain of cores we partition $P_{k}^{s}$ into $k+2$ parts, the first being the directed path $p\left(+^{s}\right)$, the next $k$ parts being $p\left(-+{ }^{s-1}\right)$, the last part being a single edge. In any $P_{k}^{s} \rightarrow P_{\ell}^{s}$ homomorphism the first part of $P_{k}^{s}$ must not map to last $s$ edges of $P_{\ell}^{s}$ because that would make the mapping of the next part impossible. So it must be mapped identically to the first part of $P_{\ell}^{s}$ and then the next $k$ parts of $P_{k}^{s}$ must also map identically to the next $k$ parts of $P_{\ell}^{s}$. This only works if $\ell \geq k$. But if $\ell>k$ the last edge of $P_{k}^{s}$ cannot be mapped anywhere. So we must have $k=\ell$ and the homomorphism must be the identity.

To see that $D_{s}$ is also a core it is enough to note that it is acyclic and has a directed Hamiltonian path. Let us denote the vertices along this path by $v_{0}, \ldots, v_{s}$.

We show $P_{k}^{s} \nrightarrow \rightarrow D_{s}$ similarly to the antichain property. Indeed, the first part of $P_{k}^{s}$ (forming a directed path) has a single homomorphism to $D_{s}$ ending at $v_{s}$. Each of the next $k$ parts must map to the path $v_{s} v_{1} v_{2} \cdots v_{s}$. But as $v_{s}$ is a sink, this homomorphism cannot be extended to the last edge of $P_{k}^{s}$.

Let $G$ be an arbitrary graph. By the statement in the last paragraph we cannot have $P_{k}^{s} \rightarrow G \rightarrow D_{s}$ for any $k \geq 0$. So it remains to prove that either $P_{k}^{s} \rightarrow G$ for some $k \geq 0$ or we have $G \rightarrow D_{s}$.

We call a vertex $v \in V(G)$ type $i$ for $0 \leq i \leq s$ if it is the image under a homomorphism of the last vertex of a path $p\left(+\left(++^{s}-1-\right)^{k}+^{i}\right)$ for some $k \geq 0$. Note that for $i \geq 1$ a type $i$ vertex is the image of the last vertex of the path $p\left(+{ }^{i}\right)$ so it is also a type $i-1$ vertex.

If there is a type $s$ vertex in $G$ we clearly have $P_{k}^{s} \rightarrow G$ for some $k \geq 0$ and we are done.

If there is no type $s$ vertex in $G$ we define $\phi: V(G) \rightarrow V\left(D_{s}\right)$ by setting $\phi(v)=v_{0}$ if $v$ is not type 0 and for $1 \leq i \leq s$ setting $\phi(v)=v_{i}$ if $v$ is not type $i$ but $v$ is type $i-1$.

We claim that $\phi$ is a $G \rightarrow D_{s}$ homomorphism.

Let $(u, v)$ be an edge of $G$. This makes $v$ the endpoint of an edge, so it is type 0 . Moreover, if $u$ is type $i$, then the path $p\left(+\left(+^{s-1}-\right)^{k}+^{i}\right)$ mapping to $G$ and ending at $u$ can be extended by the $(u, v)$ edge, making $v$ a type $i+1$ vertex. Thus if $\phi(u)=v_{j}$ and $\phi(v)=v_{j^{\prime}}$ we must have $j<j^{\prime}$. It remains to prove that $\phi(u)=v_{0}$ and $\phi(v)=v_{s}$ is impossible. Indeed, $\phi(v)=v_{s}$ implies $v$ is type $s-1$, so it is the image of the last vertex of a path $p\left(+\left(++^{s-1}-\right)^{k}+{ }^{s-1}\right)$. Extending 
this with the $(u, v)$ edge we get that $u$ is the image of the last vertex of the path $p\left(+\left(+{ }^{s-1}-\right)^{k+1}\right)$ making $u$ a type 0 vertex. This finishes the proof.

\subsection{Regularity}

From the two examples considered so far one can notice the relevance of regular languages. Indeed, while the family of words $\left\{+^{s}\left(-+^{s-1}\right)^{k}+\mid k \geq 0\right\}$ is a regular language for any $s$, the family $\left.\left\{+(+-)^{k}\right)^{k}++\mid k \geq 1\right\}$ or any of its infinite subfamilies are not regular. This connection was the basis of our upcoming paper [3] that establishes regularity as a necessary and sufficient condition for having a finite dual in this case. We state the following easy observation regarding regularity to further motivate this connection.

Lemma 3.4. Let $G$ be an arbitrary graph. The set $\left\{x \in\{+,-\}^{*} \mid p(x) \rightarrow G\right\}$ is a regular language.

Proof. We turn the graph $G$ into a nondeterministic finite automaton. The states of the automaton are the vertices of $G$ and each state is an initial and also a terminal state. For each edge $(u, v)$ of $G$ we make the transition from $u$ to $v$ possible for the letter + and the transition from $v$ to $u$ possible for the letter - . It is straightforward to see that this automaton accepts the language in the lemma.

\section{Antichains of trees}

In this section we give infinite-finite antichain duality pairs where the infinite side has trees that are not paths. The following lemma is instructive for this.

Lemma 4.1. Let $(\mathcal{A}, \mathcal{D})$ be a duality pair. Let us modify each $A \in \mathcal{A}$ by enriching it with new vertices and edges: from each sink of $A$ we start a new edge to a separate new vertex. Let $\mathcal{A}^{\prime}$ be the family of these modified graphs. Let us modify each graph $D \in \mathcal{D}$ by adding a single new vertex and edges to this vertex from every vertex of $D$. Let $\mathcal{D}^{\prime}$ be the family of these modified graphs. Then $\left(\mathcal{A}^{\prime}, \mathcal{D}^{\prime}\right)$ is a duality pair. If $\mathcal{A}$ is an antichain so is $\mathcal{A}^{\prime}$, if $\mathcal{D}$ is an antichain, so is $\mathcal{D}^{\prime}$, furthermore if $\mathcal{A}$ and $\mathcal{D}$ consist of cores so do $\mathcal{A}^{\prime}$ and $\mathcal{D}^{\prime}$.

Proof. Let $A^{\prime} \in \mathcal{A}^{\prime}$ be the modification of $A \in \mathcal{A}$ and $D^{\prime} \in \mathcal{D}^{\prime}$ be the modification of $D \in \mathcal{D}$. If we have a homomorphism $f: A^{\prime} \rightarrow D^{\prime}$, then its restriction to $A$ must map to $D$ as the single vertex of $D^{\prime} \backslash D$ is a sink in $D^{\prime}$, but no vertex of $A$ is a sink in $A^{\prime}$. But the existence of an $A \rightarrow D$ homomorphism contradicts the fact that $(\mathcal{A}, \mathcal{D})$ is a duality pair.

Let $G^{\prime}$ be an arbitrary graph. We cannot have $A^{\prime} \rightarrow G^{\prime} \rightarrow D^{\prime}$ for some $A^{\prime} \in \mathcal{A}^{\prime}$ and $D^{\prime} \in \mathcal{D}^{\prime}$ by the previous paragraph. It remains to show that $A^{\prime} \rightarrow G^{\prime}$ for some $A^{\prime} \in \mathcal{A}^{\prime}$ or $G^{\prime} \rightarrow D^{\prime}$ for some $D^{\prime} \in \mathcal{D}^{\prime}$.

Let $G$ be the subgraph of $G^{\prime}$ induced by the non-sink vertices. As $(\mathcal{A}, \mathcal{D})$ is a duality pair we either have $A \rightarrow G$ for some $A \in \mathcal{A}$ or $G \rightarrow D$ for some $D \in \mathcal{D}$. In the former case we can extend the homomorphism $A \rightarrow G$ to a homomorphism 
$A^{\prime} \rightarrow G^{\prime}$, where $A^{\prime} \in \mathcal{A}^{\prime}$ is the modified version of $A$. In the latter case we can extend the homomorphism $G \rightarrow D$ to a homomorphism $G^{\prime} \rightarrow D^{\prime}$, where $D^{\prime} \in \mathcal{D}^{\prime}$ is the modified version of $D$, by sending all vertices of $G^{\prime} \backslash G$ to the single vertex in $D^{\prime} \backslash D$.

To see that the antichain and core properties are inherited to the modified sets consider two graphs $X$ and $Y$ from the same family $\mathcal{A}$ or $\mathcal{D}$ and their modifications $X^{\prime}$ and $Y^{\prime}$. Restricting a homomorphism $X^{\prime} \rightarrow Y^{\prime}$ to $X$ we get a homomorphism $X \rightarrow Y$. Indeed, all vertices in $Y^{\prime} \backslash Y$ are sinks and no vertex in $X$ is sink in $X^{\prime}$. So if the family was antichain, then $X=Y$ and so the modified family is also an antichain. If $X=Y$ is a core, then the $X \rightarrow Y$ homomorphism must be an isomorphism and it is easy to see that the original $X^{\prime} \rightarrow Y^{\prime}$ homomorphism must also be an isomorphism.

Applying this lemma (possibly several times) to our earlier examples of infinitefinite antichain duality pairs we get several new such examples. Although the graphs on the left side of these pairs are no longer paths, they are still very similar to paths in structure.

The examples in the next lemma show better the complexity that families with a finite dual can exhibit.

Let us consider the family $T_{0}$ of all finite rooted (undirected) binary trees satisfying that each vertex is either a leaf (it has no children) or it has two children: a left child and a right child. Although these are "trees" of some kind, they are not in the category of finite directed graphs we study here and we will not apply homomorphisms to these binary trees. To emphasize the difference we denote these binary trees by lower case letters as opposed to using capitals for directed graphs. Note that the smallest member of $T_{0}$ has a single vertex.

Let $x, y, s, z \in\{+,-\}^{*}$ be words. We define the family of oriented trees $T(x, y, s, z)=\left\{t(x, y, s, z) \mid t \in T_{0}\right\}$, where $t(x, y, s, z)$ is an oriented tree obtained from $t$ by

(A) replacing each edge connecting a vertex $u$ to its left child $v$ by a copy of $p(x)$ from $u$ to $v$,

(B) replacing each edge connecting a vertex $u$ to its right child $w$ by a copy of $p(y)$ from $u$ to $w$,

(C) adding a path $p(s)$ from each leaf vertex of $t$ and

(D) adding a path $p(z)$ from the root of $t$.

Let $G_{1}$ and $G_{2}$ be the graphs depicted on Figures 1 and 2, furthermore let

$$
\mathcal{T}_{1}=T(+-,-+,--,++) \quad \text { and } \quad \mathcal{T}_{2}=T(+--,-+-,--,+++) .
$$

Theorem 4.2. (i) $\left(\mathcal{T}_{1},\left\{G_{1}\right\}\right)$ is a duality pair of core graphs. 


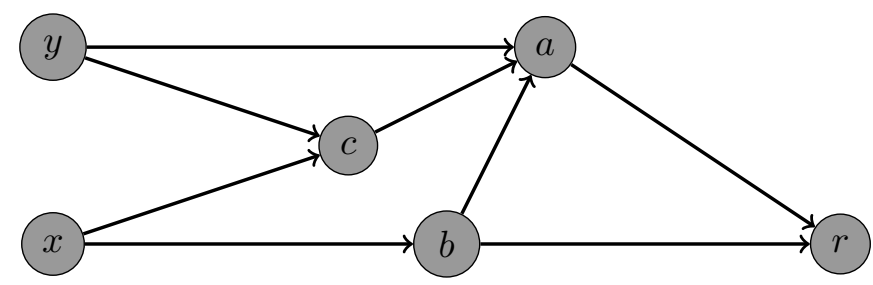

Figure 1: The graph $G_{1}$.

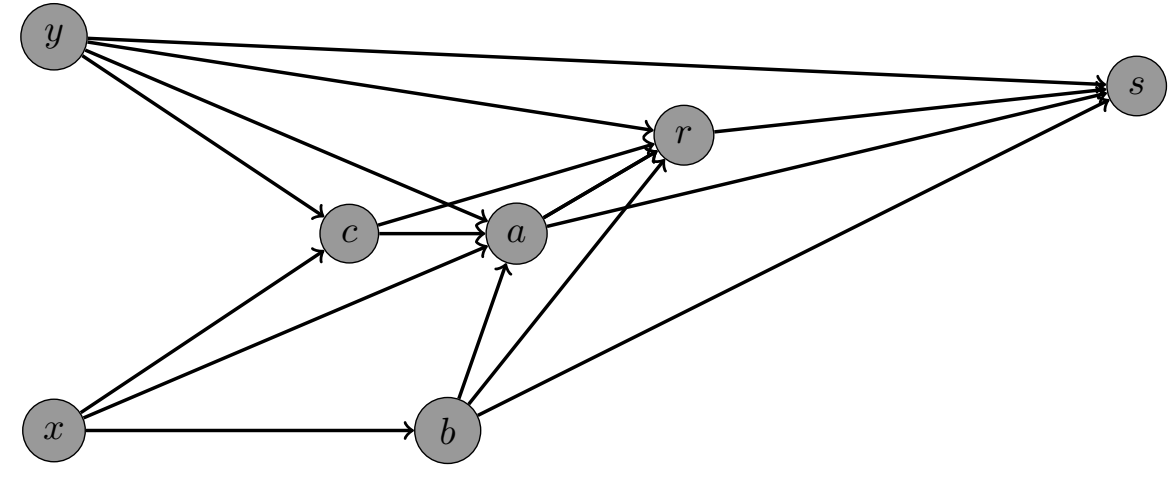

Figure 2: The graph $G_{2}$.

(ii) $\left(\mathcal{T}_{2},\left\{G_{2}\right\}\right)$ is an antichain duality pair of core graphs.

Proof. We leave the simple proofs that all involved graphs are cores and that $\mathcal{T}_{2}$ is an antichain to the diligent reader. Note that $\mathcal{T}_{1}$ is not an antichain: if $t \in T_{0}$ and $t^{\prime}$ is subtree of $t$ containing the root, then $t(+-,-+,--,++) \rightarrow$ $t^{\prime}(+-,-+,--,++)$. As a result (once (i) is proved) one also has that $\left(\left\{t_{k}(+-\right.\right.$, $\left.-+,--,++) \mid k \geq 100\},\left\{G_{1}\right\}\right)$ is a duality pair if $t_{k} \in T_{0}$ is the depth $k$ full binary tree with $2^{k}$ leaves.

Assume for a contradiction that $f: t(+-,-+,--,++) \rightarrow G_{1}$. We claim that all vertices of $t$ must map to the vertices $a$ or $r$. This is certainly true for the leaves because of the attached paths $p(--)$ could not map to $G_{1}$ otherwise. Working with a bottom up induction assume that both the left and the right children of the vertex $u$ map to $a$ or $r$. In this case the paths from $u$ to its children must be mapped to $G_{1}$ from $f(u)$ to either $a$ or $r$. Then we must have $f(u)=a$ as from no other vertex of $G_{1}$ is there both a path $p(+-)$ and a path $p(-+)$ to either $a$ or $r$. So the root vertex must also be mapped to $a$ or $r$ and the contradiction comes from there being no place in $G_{1}$ for the path $p(++)$ attached to the root.

For (i) it is left to prove that for any graph $X$ we either have $A \rightarrow X$ for an $A \in \mathcal{T}_{1}$ or we have $X \rightarrow G_{1}$. For this we define the "level" $L_{i} \subseteq V(X)$ to consist of the vertices of $X$ with a homomorphism $P_{i} \rightarrow X$ ending at $u$ but no homomorphism $P_{i+1} \rightarrow X$ ending at $u$. Here $P_{i}=p\left(+{ }^{i}\right)$ is the directed path of $i$ edges and the levels $L_{0}, L_{1}, L_{2}$ and $L_{3}$ partition $V(x)$ or we have $P_{4} \rightarrow X$ and we are done since $P_{4} \in \mathcal{T}_{1}$. 
We construct the map $\phi: V(X) \rightarrow V\left(G_{1}\right)$ as follows:

(1) Set $\phi(u)=a$ for any vertex $u \in L_{1}$ that has a $t \in T_{0}$ and a homomorphism $f: t(+-,-+,--, \epsilon) \rightarrow X$ mapping the root of $t$ to $a$. Here $\epsilon$ stands for the empty word.

(2) Set $\phi(u)=b$ for any vertex $u \in L_{1}$ not yet mapped to $a$ that either has an edge $(u, v)$ to a vertex $v \in L_{3}$ or two edges $(u, v)$ and $(w, v)$ with $w \in L_{1}$ already mapped to $a$.

(3) Set $\phi(u)=c$ for the remaining vertices $u \in L_{1}$.

(4) Set $\phi(u)=x$ if $u \in L_{0}$ and there exists an edge $(u, v)$ with $\phi(v)=b$.

(5) Set $\phi(u)=y$ for the remaining vertices $u \in L_{0}$.

(6) Set $\phi(u)=a$ if $u \in L_{2}$ and there is no $v \in L_{1}$ with $\phi(u)=a$ and $(v, u)$ an edge.

(7) Set $\phi(u)=r$ for all remaining vertices in $V(X)$.

If $\phi$ is a homomorphism $X \rightarrow G_{1}$ we are done. Otherwise one of the steps above made an edge in $X$ map outside $X$.

Steps 1-3 map the independent set $L_{1}$ so they caused no problem. Step 4 can create a problem if a vertex $u \in L_{0}$ has edges $(u, v)$ and $(u, w)$ with $v, w \in L_{1}$, $\phi(v)=b$ and $\phi(w)=a$. But in this case the homomorphisms triggering $\phi(v)=b$ and $\phi(w)=a$ in steps 2 and 1 can be combined (together with the $w u v$ path) with a homomorphism triggering $\phi(v)=a$ in the first step, a contradiction.

Step 5 cannot cause trouble as both $(y, a)$ and $(y, c)$ are edges in $G_{1}$.

Steps 6 or 7 cause trouble if there is a vertex $u \in L_{2} \cup L_{3}$ with $(v, u)$ an edge from a vertex $v \in L_{0}$ with $\phi(v)=x$. But then there is a vertex $w \in L_{1}$ with $(v, w)$ an edge and $\phi(w)=b$. Here again, the homomorphism of $p(++-+)$ to $X$ ending in the vertices $u v w$ can be combined to the homomorphism triggering $\phi(w)=b$ to obtain a homomorphism triggering $\phi(w)=a$, a contradiction.

Finally in step 7 we can map both ends of an $(u, v)$ edge to $r$. This happens if there exists an edge $(w, u)$ from a vertex $w \in L_{1}$ with $\phi(w)=a$. This may indeed happen, but then the homomorphism triggering $\phi(w)=a$ can be combined to the directed path wuv to get $A \rightarrow X$ for a tree $A \in \mathcal{T}_{1}$. This finishes the proof of part (i).

(ii) The proof of this part is only slightly more complicated.

Assume for a contradiction that $f: t(+--,-+-,--,+++) \rightarrow G_{2}$. We claim that all vertices of $t$ must map to the vertices $a, r$ or $s$. This can be shown exactly like the corresponding statement in part (i). So the root vertex must also be mapped to $a$ or $u$ and the contradiction comes from there being no place in $G_{2}$ for the path $p(+++)$ attached to the root. 
Finally we assume $X$ is graph with no $A \rightarrow X$ homomorphism for any $A \in \mathcal{T}_{2}$. We construct the homomorphism $\phi: X \rightarrow G_{2}$ similarly to part (i). We partition $V(X)$ into levels $L_{i}$ as we did above. As $P_{5} \in \mathcal{T}_{2}$ does not map to $X, V(X)$ is partitioned into the sets $L_{0}, L_{1}, L_{2}, L_{3}$ and $L_{4}$.

(1) Set $\phi(u)=a$ for any vertex $u \in L_{1}$ that has a $t \in T_{0}$ and a homomorphism $f: t(+--,-+-,--, \epsilon) \rightarrow X$ mapping the root of $t$ to $a$.

(2) Set $\phi(u)=b$ for any vertex $u \in L_{1}$ not yet mapped to $a$ that has either a homomorphism mapping $p(+----)$ to $G_{2}$ from $u$ or a homomorphism of $p(+--)$ to $G_{2}$ from $u$ to a vertex $v \in L_{1}$ with $\phi(v)=a$.

(3) Set $\phi(u)=c$ for the remaining vertices $u \in L_{1}$.

(4) Set $\phi(u)=x$ if $u \in L_{0}$ and there exists an edge $(u, v)$ with $\phi(u)=b$.

(5) Set $\phi(u)=y$ for the remaining vertices $u \in L_{0}$.

(6) Set $\phi(u)=a$ if $u \in L_{2}$ and there is no $v \in L_{1}$ with $\phi(u)=a$ and $(v, u)$ an edge.

(7) Set $\phi(u)=r$ for all remaining vertices $u \in L_{2}$.

(8) Also set $\phi(u)=r$ for vertices $u \in L_{3}$ with no edge $(v, u)$ from a vertex $v \in L_{2}$ with $\phi(v)=r$.

(9) Set $\phi(u)=s$ for all remaining vertices $u \in V(X)$.

The proof that $\phi$ is indeed a homomorphism is almost identical to the corresponding argument in part (i).

We finish the paper with a simple observation that shows how to combine duality pairs to obtain new pairs with several graphs on the right side. For simplicity we restrict attention to combining two duality pairs with single graphs on the right hand side that are incomparable.

In the following proposition and example $A_{1} \cup A_{2}$ denotes the disjoint union of the graphs $A_{1}$ and $A_{2}$.

Proposition 4.3. Let $\left(\mathcal{A}_{1}, \mathcal{D}_{1}\right)$ and $\left(\mathcal{A}_{2}, \mathcal{D}_{2}\right)$ be duality pairs and let us partition $\mathcal{A}_{i}$ into $\mathcal{A}_{i}^{\prime}=\left\{A \in \mathcal{A}_{i} \mid \exists B \in \mathcal{A}_{3-i}: B \rightarrow A\right\}$ and $\mathcal{A}_{i}^{\prime \prime}=\mathcal{A}_{i} \backslash \mathcal{A}_{i}^{\prime}$ for $i=1,2$.

(i) $(\mathcal{A}, \mathcal{D})$ is a duality pair, where $\mathcal{D}=\mathcal{D}_{1} \cup \mathcal{D}_{2}$ and $\mathcal{A}=\left\{A_{1} \cup A_{2} \mid A_{1} \in\right.$ $\left.\mathcal{A}_{1}, A_{2} \in \mathcal{A}_{2}\right\}$.

(ii) $\left(\mathcal{A}^{\prime}, \mathcal{D}\right)$ is also a duality pair, where $\mathcal{A}^{\prime}=\mathcal{A}_{1}^{\prime} \cup \mathcal{A}_{2}^{\prime} \bigcup\left\{A_{1} \cup A_{2} \mid A_{1} \in\right.$ $\left.\mathcal{A}_{1}^{\prime \prime}, A_{2} \in \mathcal{A}_{2}^{\prime \prime}\right\}$. 
(iii) If each $\mathcal{A}_{i}$ is an antichain and $\left|\mathcal{D}_{1}\right|=\left|\mathcal{D}_{2}\right|=1$, then $\mathcal{A}^{\prime}$ can be made an antichain by removing possible duplicates: leaving one member only from each equivalent pair of graphs from $\mathcal{A}_{1}$ and $\mathcal{A}_{2}$.

Proof. For (i) it is enough to note that $A_{1} \cup A_{2} \rightarrow B$ if and only if $A_{1} \rightarrow B$ and $A_{2} \rightarrow B$.

For (ii) take $A_{2} \in \mathcal{A}_{2}^{\prime}$ and a graph $A_{1} \in \mathcal{A}_{1}$ with $A_{1} \rightarrow A_{2}$. As $A_{1} \cup A_{2}$ is equivalent to $A_{2}$ we can put $A_{2}$ into the left side of the duality pair $(\mathcal{A}, \mathcal{D})$. But then all graphs $A_{1}^{\prime} \cup A_{2}$ can be removed from there as $A_{2}$ maps to these graphs. Doing this for all $A_{2} \in \mathcal{A}_{2}^{\prime}$ and similar changes for the graphs in $\mathcal{A}_{1}^{\prime}$ one obtains $\mathcal{A}^{\prime}$ and (ii) is proved.

To prove (iii) take $A \in \mathcal{A}^{\prime}$ and consider the sets $S_{i}(A)=\left\{B \in \mathcal{A}_{i} \mid B \rightarrow A\right\}$ for $i=1,2$. For a graph $A=A_{1} \cup A_{2}$ with $A_{1} \in \mathcal{A}_{1}^{\prime \prime}, A_{2} \in \mathcal{A}_{2}^{\prime \prime}$ we have $S_{1}(A)=\left\{A_{1}\right\}$ and $S_{2}(A)=\left\{A_{2}\right\}$ since the graphs in $\mathcal{A}_{1} \cup \mathcal{A}_{2}$ are connected (Lemma 2.2), thus they map to $A$ if and only if they map to $A_{1}$ or $A_{2}$. For $A \in \mathcal{A}_{i}^{\prime}$ and $i=1$ or 2 we have $S_{i}(A)=\{A\}$. Since $A \rightarrow A^{\prime}$ implies $S_{i}(A) \subseteq S_{i}\left(A^{\prime}\right)$ for $A, A^{\prime} \in \mathcal{A}^{\prime}$ and $i=1,2$ the only possibility of such a map with $A \neq A^{\prime}$ is $A \in \mathcal{A}_{i}^{\prime}$ and $A^{\prime} \in \mathcal{A}_{3-i}^{\prime}$. From $A \in \mathcal{A}_{i}^{\prime}$ we have $A^{\prime \prime} \in \mathcal{A}_{3-i}$ with $A^{\prime \prime} \rightarrow A \rightarrow A^{\prime}$. As $\mathcal{A}_{3-i}$ is an antichain we must have $A^{\prime \prime}=A$ and thus $A$ and $A^{\prime}$ are equivalent.

We can apply this lemma to combine any two of the several examples of infinite-finite duality pairs in this paper or even one such example with a simple duality with a single tree on the left hand side. We chose the duality pairs $\left(\left\{P_{k}^{4} \mid k \geq 0\right\},\left\{D_{4}\right\}\right)$ from Example 3.3 and $\left(\mathcal{T}_{2},\left\{G_{2}\right\}\right)$ from Theorem 4.2. Note that $P_{0}^{4}$ is the directed path with five edges and it appears in $\mathcal{T}_{2}$ but no homomorphism exist from a member of $\mathcal{T}_{2}$ to some $P_{k}^{4}$ with $k \geq 1$ or vice versa. Thus from Lemma 4.3 we get the following

Example 4.4. The following is an antichain duality pair of core graphs:

$$
\left(\left\{P_{0}^{4}\right\} \cup\left\{P_{k}^{4} \cup A \mid k \geq 1, A \in \mathcal{T}_{2} \backslash\left\{P_{0}^{4}\right\}\right\},\left\{D_{4}, G_{2}\right\}\right) .
$$

\section{References}

[1] D. Duffus, P.L. Erdős, J. Nešetřil, L. Soukup, Antichains in the homomorphism order of graphs, Comment Math. Univ. Carolinae 48 (4) (2007), 571583.

[2] P.L. Erdős, L. Soukup, No finite-infinite antichain duality in the homomorphism poset of directed graphs, Order 27 (3) (2010), 317-325.

[3] P.L. Erdős, C. Tardif, G. Tardos, Caterpillar dualities and regular languages, manuscript (2012).

[4] P.L. Erdős, D. Pálvölgyi, C. Tardif, G. Tardos, On infinite-finite tree-duality pairs of relational structures, manuscript (2012). 
[5] J. Foniok, J. Nešetřil, C. Tardif, Generalized dualities and maximal finite antichains in the homomorphism order of relational structures, Europ. J. Comb 29 (2008), 881-899.

[6] J. Nešetřil, A. Pultr, On classes of relations and graphs determined by subobjects and factorobjects, Discrete Math. 22 (1978), 287-300.

[7] J. Nešetřil, C. Tardif, Duality theorems for finite structures (characterising gaps and good characterisations) J. Comb. Theory Ser. (B) 80 (2000), 80-97 